\title{
ARTÍCULOS
}

\section{Propuesta de un índice de diversidad funcional. Aplicación a un bosque semideciduo micrófilo de Cuba Oriental}

\author{
Proposal of an index for functional diversity. \\ Application to a microphyll semi-deciduous forest in eastern Cuba
}

\author{
Arturo Salmerón López ${ }^{\text {a*}, ~ G r e t e l ~ G e a d a ~ L o ́ p e z ~}{ }^{\text {b }}$ María del Carmen Fagilde Espinoza a \\ *Autor de correspondencia: a Museo de Historia Natural Tomás Romay, Centro Oriental de Ecosistemas y Biodiversidad, \\ Calle José A. Saco, N. 601, Santiago de Cuba, Cuba, tel.: +5322 626568, arturo@bioeco.cu \\ ${ }^{\text {b } U n i v e r s i d a d ~ d e ~ P i n a r ~ d e l ~ R i ́ o, ~ C e n t r o ~ d e ~ E s t u d i o s ~ F o r e s t a l e s, ~ C u b a . ~}$
}

\begin{abstract}
SUMMARY
Functional diversity has emerged as a relevant aspect in the ecosystem studies; several indexes have been used for its evaluation. However, these indexes have not considered the functional redundancy among species, even when its implication in forest resilience facing natural and anthropogenic disturbances have been recognized. In this study an index for functional diversity, considering functional redundancy, is proposed and applied to know changes in functional diversity types according to its disturbance responses, in sites under different disturbance levels, in a microphyll semi-deciduous forest. The probability theory was used to derive a functional biodiversity index from the Simpson biodiversity index. The sensitivity of the index in relation to changes in the individual, species and functional type number was analyzed and compared with other indexes proposed in literature. The less functional diversity value was found in highly disturbed sites, and the highest value in the less disturbed sites. Results confirmed that in less disturbed sites, individuals were more uniformly distributed inside the species and these inside the functional types, than those individuals found in highly disturbed areas; it confers the system a superior functional redundancy. It is concluded that the proposed index is, besides, a good reference of the resilience in a forest ecosystem, and the forest management oriented to keep the functional diversity, as it is interpreted, is a guaranty for the conservation of the forest resilience and integrity.
\end{abstract}

Key words: functional types, redundancy, forest resilience.

\section{RESUMEN}

La diversidad funcional ha emergido como un aspecto de relevancia en el estudio de los ecosistemas; varios índices han sido empleados para evaluarla, pero estos no han considerado la redundancia funcional entre especies aun cuando su implicación en la resiliencia de los bosques frente a perturbaciones naturales y antrópicas ha sido reconocida. En este estudio se propuso un índice para evaluar la diversidad funcional que considera dicha redundancia y se aplicó para conocer los cambios en la diversidad de tipos funcionales según las respuestas a perturbaciones, en sitios sometidos a diferentes niveles de antropización en un bosque semideciduo micrófilo. El índice fue obtenido mediante análisis probabilísticos a partir del índice de Simpson de biodiversidad. Se analizó su sensibilidad a cambios en los números de individuos, especies y tipos funcionales, y se comparó con otros propuestos en la literatura. El menor valor de diversidad funcional se encontró en los sitios muy perturbados y el mayor en los sitios poco perturbados. Los resultados confirmaron que en los sitios menos perturbados los individuos estaban más uniformemente distribuidos en las especies y estas en los tipos funcionales, lo que le confiere al bosque mayor redundancia funcional. El índice propuesto es, además, un buen referente de la resiliencia del sistema, por lo que el manejo del bosque orientado a mantener la diversidad funcional medida según el índice, es una garantía para la conservación de la resiliencia y la integridad del bosque.

Palabras clave: tipos funcionales, redundancia, resiliencia.

\section{INTRODUCCIÓN}

La diversidad funcional ha emergido como un aspecto de importancia crucial en el estudio de los procesos ecosistémicos y en la actualidad existe consenso respecto a que el efecto de la diversidad en los ecosistemas, se debe más a los rasgos funcionales de las especies que al número de especies per se (Díaz y Cabido 2001, Quétier et al. 2008, Petchey y Gaston 2009, Thompson et al. 2009, Díaz et al. 2011, Gillison et al. 2013). La diversidad funcional se re- 
fiere al valor, rango y abundancia relativa de los rasgos funcionales de los organismos presentes en un ecosistema (Díaz y Cabido 2001, Gillison et al. 2013).

Parte del borde costero sur de Cuba Oriental se encuentra cubierto por un bosque semideciduo micrófilo que ha sido reconocido como una de las prioridades para la conservación y uso sostenible en la Reserva de la Biosfera Baconao ${ }^{1}$. Salmerón et al. (2016), trabajando en sitios poco, medianamente y muy perturbados de una de sus zonas núcleo de conservación (Reserva Ecológica Siboney Juticí), determinan la existencia en este bosque de tres tipos funcionales de respuestas a las perturbaciones, a los que denominan: cobertoras, colonizadoras y estabilizadoras.

Las perturbaciones antrópicas ocurridas ocasionan cambios diferentes en la composición y abundancia de esos tipos funcionales (Salmerón et al. 2016), alterando a su vez la capacidad de recuperación del bosque al cesar los disturbios. Los índices de biodiversidad comúnmente usados en la literatura, no son capaces de captar completamente esta información. Por lo tanto, se necesita un índice que permita evaluar cuantitativamente los cambios producidos en la composición y abundancia de los tipos funcionales (índice de diversidad funcional) bajo diferentes perturbaciones antrópicas y a su vez posibilite guiar, de manera eficiente, los esfuerzos de restauración para el logro de estados con mayor resiliencia frente al régimen de perturbaciones naturales y humanas verificado en el área.

Entre los índices más usados para evaluar diferentes aspectos de la diversidad biológica (Moreno 2001, Feinsinger 2004) se encuentra el de Shannon - Weaver (H), el de dominancia de Simpson $(\lambda)$ y el inverso de $\operatorname{Simpson}\left(\mathrm{C}_{\text {inv }}\right)$.

El índice de Simpson es un índice de dominancia más que de diversidad y representa la probabilidad de que dos individuos escogidos al azar pertenezcan a la misma especie (Moreno 2001). Su inverso se considera como un buen indicador de diversidad (Feinsinger 2004). Varios estudios relacionan la riqueza y la diversidad de especies con los niveles de perturbación en ecosistemas boscosos (Mishra et al. 2004, Kumar y Ram 2005, Sahu et al. 2008).

A pesar de la abundante información sobre métodos para evaluar la diversidad funcional (Mason et al. 2003, Leps et al. 2006, Mouchet et al. 2010, Casanoves et al. 2011), la mayoría de los estudios se limitan a la caracterización de rasgos funcionales y cuando más definen tipos funcionales de plantas, (Lavorel 1999, Gondard et al. 2003, Kooyman y Rossetto 2008, Uriarte et al. 2010, Fyllas et al. 2012) entendidos estos como conjuntos de especies que muestran respuestas similares al ambiente y efectos análogos en el funcionamiento de los ecosistemas, sin realizar análisis de la diversidad funcional.

Para varios autores (Mason et al. 2003, Leps et al. 2006, Mouchet et al. 2010, Casanoves et al. 2011) un índi-

\footnotetext{
Salmerón A, LO Alvarez. 2013. Plan de manejo para el Área Protegida de Recursos Manejados "Reserva de la Biosfera Baconao". BIOECO. 178 p. No publicado.
}

ce de diversidad funcional debe ser insensible al método de construcción de tipos funcionales y al número de especies, y no variar cuando una especie es sustituida por otras con el mismo valor funcional y cuyos números de individuos sumados equivalen al número de individuos de la especie desaparecida. No obstante, varios estudios determinan la existencia de tipos funcionales y consideran la existencia de varias especies dentro de cada tipo (Petchey y Gaston 2009, Fyllas et al. 2012, Gillison et al. 2013)

La mayoría de los autores plantean varios criterios a tener en cuenta cuando se usa un indice para evaluar la diversidad funcional (Mason et al. 2003, Leps et al. 2006 y 2011, Mouchet et al. 2010) el índice debe considerar la abundancia de las especies en los tipos funcionales en vez del número de especies, asimismo el mismo debe permanecer constante cuando una especie es sustituida por otras dos con rasgos funcionales similares y cuya suma de individuos es equivalente al número de individuos de la especie sustituida. Para Mason et al. (2003) un índice de diversidad funcional debe ser invariable al método de definición de los tipos funcionales.

Los índices para evaluar la diversidad funcional tienen un potencial extraordinario para revelar los procesos que estructuran las comunidades biológicas y son agrupados en tres categorías, cada una de las cuales responde a uno de los siguientes aspectos: riqueza funcional, uniformidad funcional y divergencia funcional. La uniformidad funcional se refiere a cuan regularmente la abundancia de las especies está distribuida en el espacio funcional (Mouchet et al. 2010).

El índice de Rao de biodiversidad, adaptado para la diversidad funcional mediante el uso de rasgos funcionales para evaluar variabilidad, es una aproximación del índice de Simpsom para diversidad (Leps et al. 2006). Si la proporción de especies $i$ dentro de una comunidad es $\mathrm{p}_{\mathrm{i}}$ y la diferencia entre las especies $i$ y $j$ es $\mathrm{d}_{\mathrm{ij}}$, entonces el coeficiente de Rao es:

$$
F D=\sum_{i=1}^{s} \sum_{j=1}^{s} d_{i j} p_{i} p_{j}
$$

donde: $s=$ número de especies y $d_{i j}$ varía de cero (todas las especies tienen absolutamente el mismo valor del rasgo funcional analizado) a uno (las especies tienen rasgos funcionales completamente diferentes).

Casanoves et al. (2011) realizan un breve análisis de varios índices, en particular del índice MFAD:

$$
M F A D=\frac{\sum_{i=1}^{N} \sum_{j=1}^{N} d_{i j}}{N}
$$

donde: $\mathrm{N}=$ número de unidades funcionales resultante de la combinación de especies con valores similares en los rasgos funcionales analizados. Aquí, el índice clasifica a 
las especies en unidades (tipos) funcionales, sin diferenciar entre estas al interior del tipo funcional. Ahora $d_{i j}=$ diferencia entre los tipos $i$ y $j$.

Dos o más especies son consideradas redundantes cuando pueden ser ubicadas dentro de un mismo tipo funcional con respecto a un proceso del ecosistema. La desaparición de una o más de esas especies no afecta ese proceso del ecosistema significativamente (Díaz y Cabido 2001).

En un ecosistema sometido a perturbaciones, la probabilidad de que algunas de las especies sobrevivientes a los cambios mantengan las propiedades del sistema, será mayor mientras más grande sea el número de especies funcionalmente similares (Díaz y Cabido 2001). La redundancia incrementa la posibilidad del sistema de mantener su funcionamiento (y las interacciones entre los tipos funcionales) en caso de pérdidas de especies (Thompson et al. 2009).

La resiliencia de los ecosistemas, entendida como la capacidad del sistema de retornar a su estado previo, o muy cercano a este, después de experimentar una perturbación dada (Gunderson 2000), está directamente relacionada con la redundancia funcional (Thompson et al. 2009). El manejo de los ecosistemas dirigido al mantenimiento de su redundancia funcional, es una garantía para el mantenimiento de la complejidad estructural ecosistémica (Petchey y Gaston 2009) y por lo tanto para asegurar la integridad ecológica de los mismos. Lo anterior refuerza la necesidad de considerar la redundancia de las especies al evaluar la diversidad funcional.

Si se considera la relación entre la resiliencia del sistema y su redundancia funcional (Gunderson 2000, Thompson et al.2009) se supone entonces que un índice de diversidad funcional capaz de captar la redundancia al interior de los tipos funcionales, podrá aportar información no solo sobre los cambios en la diversidad funcional en un sistema perturbado, sino también sobre los cambios en su resiliencia frente a dichas perturbaciones.

Basado en la anterior hipótesis, el presente estudio tiene como objetivo proponer y validar un índice de diversidad funcional sensible a la redundancia al interior de los tipos funcionales, para evaluar los cambios ocasionados por las perturbaciones antrópicas, en la diversidad funcional y en la capacidad de recuperación del bosque semideciduo micrófilo de la Reserva Ecológica Siboney Juticí.

Aunque se aplica en una de las zonas núcleo de conservación, los resultados son válidos para todos los ecótopos de ese bosque en la Reserva de la Biosfera Baconao, que se encuentran distribuidos a lo largo de su borde costero y están sometidos a un régimen similar de perturbaciones naturales y antrópicas.

\section{MÉTODOS}

La Reserva Ecológica Siboney Juticí, es una zona núcleo de la Reserva de la Biosfera Baconao y se encuen- tra localizada en el borde costero de la región oriental de Cuba, al sureste de Santiago de Cuba. Posee una superficie total de 1.854 ha, de los cuales, aproximadamente 1.050 ha son terrestres ${ }^{2}$. El sector terrestre de la reserva está constituido fundamentalmente por calizas cuaternarias fuertemente carsificadas y escalonadas hacia el Sur en terrazas marinas. Las precipitaciones reportadas en el área no sobrepasan los $800 \mathrm{~mm}$ anuales.

En los lugares más altos de las terrazas, así como en las depresiones, se acumularon depósitos de arcillas resultantes de la decalsificación de las calizas. En esos sitios se estableció el bosque semideciduo micrófilo, cuyos ecótopos se extienden por más del $70 \%$ de la superficie terrestre de la Reserva Ecológica. Las especies más abundantes son: Adelia ricinella L., Amyris elemifera L., Banara minutiflora (A. Rich.) Sleumer, Bourreria virgata G. Don., Bursera simaruba (L.) Sargent., Citharexylum spinosum L., Coccoloba diversifolia Jacq., Colubrina elliptica (Sw.) Briz. et W. L. Stern, Cordia gerascanthus L., Coulteria linnaei (Griseb.) Acev.-Rodr., Crossopetalum rhacoma Hitchc., Croton lucidus Gage, Diospyros grisebachii (Hiern.) Standl., Erithalis fruticosa L., Erythroxylum havanense Jacq., Erythroxylum rotundifolium Lunan, Eugenia cowelli Britton et P. Wilson, Eugenia iteophylla Krug et Urb, Eugenia monticola DC., Exostema caribaeum (Jacq.) Roem. et Schult., Guettarda cueroensis Britton, Guettarda elliptica Sw., Gymnanthes lucida Sw., Heterosavia bahamensis (Britton) Petra Hoffm., Melochia tomentosa L., Picrodendron baccatum (L.) Krug et Urb., Plumeria obtusa Bert. Ex A.DC., Pseudocarpidium avicennioides (A. Rich.) Millsp., Pseudocarpidium ilicifolium (A. Rich.) Millsp., Randia aculeata L., Rauvolfia ligustrina Willd. ex Roem. et Schult., Senna atomaria (L). H. S. Irwin et Barneby, Sideroxylon salicifolium C.F. Gaertn., Tabebuia myrtifolia Britton, Tecoma stans (L.) Griseb., Thouinia trifoliata Poit., Vachellia macracantha (Humb. et Bonpl. ex Willd.) Seigler et Ebinger, Zanthoxylum fagara (L.) Sarg., Zanthoxylum pistaciifolium Griseb. (Reyes y Acosta 2005, González et al. 2013, Salmerón et al. 2016).

Índice de diversidad funcional. Se consideraron los tipos funcionales de plantas de acuerdo con los criterios de Lavorel (1999) y Díaz y Cabido (2001), asumidos por Salmerón et al (2016). Para la definición del índice de diversidad funcional se tuvo en cuenta que cada tipo funcional pudo estar integrado por varias especies con diferentes números de individuos y que el índice obtenido debió ser sensible a la variación en el número de individuos, de especies y de tipos funcionales.

Partiendo del índice de Simpsom y considerando el significado del mismo, se realizaron los procedimientos matemáticos, basados en la teoría de probabilidades, que per-

\footnotetext{
González A, M Abad, A Salmerón, L O Álvarez, M Silot. 2013. Plan de Manejo de la Reserva Ecológica Siboney Juticí, Cuba. BIOECO. 250 p. No publicado.
} 
mitieron obtener un índice que expresara una probabilidad similar a la de aquel, pero en este caso para especies y tipos funcionales. Se consideró su inverso como índice de diversidad funcional, siguiendo el criterio de Feinsinger (2004).

Para verificar la sensibilidad y el significado del índice obtenido, se analizaron datos virtuales correspondientes a una comunidad vegetal hipotética, en la que se hizo variar de manera controlada tres campos de datos (número de tipos funcionales, número de especies por tipos funcionales y número de individuos por especies) y se calculó la diversidad funcional en cada caso.

Diversidad funcional en el bosque semideciduo micrófilo. Para evaluar la diversidad funcional, se aplicó el índice de diversidad funcional propuesto en los sitios poco, medianamente y muy perturbados definidos por Salmerón et al. (2016). En cada sitio se tuvo en cuenta la riqueza y abundancia relativa por especies, considerando los tres tipos funcionales determinados previamente por esos autores. Los datos referidos a unidad muestreal y tamaño de muestra pueden verse en Salmerón et al. (2016).

Se calcularon los valores para los índices de biodiversidad comúnmente usados en la literatura, el de Shannon Weaver $(H)$, el de dominancia de Simpson $(\lambda)$ y el inverso de Simpson $\left(\mathrm{C}_{\mathrm{inv}}\right)$ (ecuaciones 3,4 y 5 ).

$$
\begin{gathered}
H=-\sum_{i=1}^{S} p_{i} \ln p_{i} \\
\lambda=\sum_{i=1}^{S} p_{i}^{2} \\
C_{i n v}=\frac{1}{\sum_{i=1}^{S} p_{i}{ }^{2}}
\end{gathered}
$$

y se compararon los valores de diversidad funcional con aquellos. Se realizó un análisis sobre el significado de las diferencias obtenidas entre los índices en sitios sometidos a diferentes niveles de perturbación y se analizó cual es la información nueva que aporta el índice de diversidad funcional utilizado sobre la capacidad de recuperación del bosque en cada caso.

\section{RESULTADOS}

Índice de diversidad funcional. Partiendo de una parcela hipotética correspondiente a un tipo de cobertura vegetal dado, donde se han listado $S$ especies, entonces para cada especie $i$ se tuvieron $\mathrm{n}_{\mathrm{i}}$ individuos, de manera que el número total $N$ de individuos en la parcela fue:

$$
N=\sum_{i=1}^{S} n_{i}
$$

Se supuso además, que las $S$ especies identificadas se encontraron distribuidas en varios tipos funcionales determinados según un criterio dado (por ejemplo, los tipos funcionales de acuerdo con la respuesta a las perturbaciones antrópicas, como en este estudio), de manera que el número de tipos funcionales fue $T$. Entonces, asignándole $S_{j}$ especies a cada tipo funcional, el número de individuos en cada tipo funcional, $m_{j}$, fue igual a la suma del número de individuos de cada una de las especies correspondientes al tipo funcional dado:

$$
m_{j}=\sum_{i=1}^{S_{j}} n_{i j}=n_{1 j}+n_{2 j}+\cdots+n_{S_{j} j}
$$

y el número total de individuos fue también igual a la suma del número de individuos presentes en cada tipo funcional:

$$
N=\sum_{j=1}^{T} m_{j}
$$

En tales condiciones, denominando $A_{i}$ y $B_{j}$ a los sucesos consistentes en que al escoger al azar un individuo este correspondiera a una de las $n_{i}$ especies (suceso $A_{i}$ ) o a uno cualquiera de los tipos funcionales (suceso $B_{j}$ ), se encontró la probabilidad de ocurrencia de tales sucesos como:

$$
P\left(A_{i}\right)=\frac{n_{i}}{N}
$$

$\mathrm{y}$

$$
P\left(B_{j}\right)=\frac{m_{j}}{N}
$$

Obviamente, la probabilidad de que al escoger dos individuos estos pertenecieran a la misma especie $i$ o al mismo tipo funcional $j$, resultó $\left(\frac{n_{i}}{N}\right)^{2} \circ\left(\frac{m_{j}}{N}\right)^{2}$, respecti-
vamente.

Luego, considerando el suceso consistente en que al elegir dos pares de individuos se obtuviera que un individuo de cada par elegido correspondiera a la misma especie $i$ (del tipo funcional $j$ ) y el otro individuo de cada par elegido correspondiera a cualquiera de las $S_{j}$ especies del tipo funcional $j$, se demostró que la probabilidad de tal suceso es:

$$
\rho_{j}=\left(\frac{m_{j}}{N}\right)^{2} \sum_{i=1}^{S_{j}}\left(\frac{n_{i j}}{N}\right)^{2}
$$

generalizando el suceso incluyéndole la posibilidad de obtener coincidencia par a par en dos individuos de la misma especie y dos individuos del mismo tipo funcional que la especie de los dos anteriores, para todos los $N$ individuos, las $S$ especies y los $T$ tipo funcionales, se obtuvo: 
$\rho=\left(\frac{m_{1}}{N}\right)^{2} \sum_{i=1}^{S_{1}}\left(\frac{n_{i 1}}{N}\right)^{2}+\left(\frac{m_{2}}{N}\right)^{2} \sum_{i=1}^{S_{2}}\left(\frac{n_{i 2}}{N}\right)^{2}+\cdots+\left(\frac{m_{j}}{N}\right)^{2} \sum_{i=1}^{S_{j}}\left(\frac{n_{i j}}{N}\right)^{2}$

que puede escribirse como:

$$
\rho=\sum_{j=1}^{T}\left(\frac{m_{j}}{N}\right)^{2} \sum_{i=1}^{S_{j}}\left(\frac{n_{i j}}{N}\right)^{2}
$$

Donde:

$T$ : número de tipos funcionales identificados

$S_{j}$ : número de especies en el tipo funcional j

$m_{j}$ : número de individuos en tipo funcional $\mathrm{j}$

$n_{i j}$ : número de individuos en la especie $\mathrm{i}$ del tipo funcional $\mathrm{j}$

$N$ : número total de individuos

El parámetro $\rho$ representa una probabilidad similar al índice de dominancia de Simpson, calculada ahora para especies y tipos funcionales.

Para evaluar la diversidad funcional, considerando que la diversidad se puede expresar como el inverso de la dominancia, se propuso el índice:

$$
\sigma=\frac{1}{\rho}
$$

Sensibilidad del índice $\sigma$ a los cambios en la abundancia relativa de las especies, al número de especies y al número de tipos funcionales. El valor mínimo obtenido para el índice $\sigma$ fue 1. Es fácil demostrar que cuando existe solo una especie, esta corresponde a un tipo funcional $(T=1)$ y toma el valor 1 para cualquier número $N$ de individuos de esa especie.

La sensibilidad del índice a las variaciones realizadas en el número de especies y de tipos funcionales se muestra en los cuadros 1 y 2 ( $\operatorname{casos} 1$ y 2 ).

En el caso 1 (cuadro 1) se mantuvo el mismo número de individuos y especies en cada subcaso (1.1, 1.2 y 1.3), pero el número de tipos funcionales se varió. El índice $\sigma$ aumentó $(4,16$ y 64) al aumentar el número de tipos funcionales. En el caso 2 (cuadro 2) se mantuvo el mismo número de individuos y tipos funcionales en cada subcaso (2.1 y 2.2), pero el número de especies se varió. El índice $\sigma$ aumentó (54 a 108) al aumentar el número de especies por tipo funcional. En ambos casos se mantuvo una distribución homogénea de individuos en especies y tipos funcionales.

En el caso 3 (cuadro 3) se mantuvo el mismo número de individuos (120) pero con una distribución heterogénea de estos en las especies y tipos funcionales. En el caso 3.1, similar al 2.1 en T, S y N, pero con una especie dominante en cada tipo funcional, la diversidad funcional decreció (54 a 48) con respecto al caso 2.1 .
Cuadro 1. Caso 1. 120 individuos igualmente distribuidos en el mismo número de especies, pero en números de tipos funcionales diferentes. T: número de tipos funcionales; S: número de especies; N: número total de individuos; $\sigma$ : diversidad funcional.

Case 1.120 individuals equally distributed in same number of species, however in different number of functional types. T: number of functional types; S: number of tree species; $\mathrm{N}$ : total individual number;

\begin{tabular}{|c|c|c|c|}
\hline Subcasos & $\begin{array}{c}\text { Tipos } \\
\text { funcionales }\end{array}$ & $\begin{array}{c}\text { Especies } \\
\text { (Individuos) }\end{array}$ & $\sigma$ \\
\hline \multirow{4}{*}{$\begin{array}{l}\text { Caso 1.1: } \mathrm{T}=1 ; \\
\mathrm{S}=4 ; \mathrm{N}=120\end{array}$} & \multirow{4}{*}{ A } & a (30) & \multirow{4}{*}{4} \\
\hline & & b (30) & \\
\hline & & c (30) & \\
\hline & & $\mathrm{d}(30)$ & \\
\hline \multirow{4}{*}{$\begin{array}{l}\text { Caso 1.2: } \mathrm{T}=2 ; \\
\mathrm{S}=4 ; \mathrm{N}=120\end{array}$} & \multirow{2}{*}{ A } & a (30) & \multirow{4}{*}{16} \\
\hline & & b (30) & \\
\hline & \multirow{2}{*}{ B } & c (30) & \\
\hline & & $\mathrm{d}(30)$ & \\
\hline \multirow{4}{*}{$\begin{array}{l}\text { Caso 1.3: } \mathrm{T}=4 ; \\
\mathrm{S}=4 ; \mathrm{N}=120\end{array}$} & A & a (30) & \multirow{4}{*}{64} \\
\hline & B & $\mathrm{b}(30)$ & \\
\hline & $\mathrm{C}$ & c (30) & \\
\hline & $\mathrm{D}$ & $\mathrm{d}(30)$ & \\
\hline
\end{tabular}
$\sigma$ : functional diversity.

Cuadro 2. Caso 2. 120 individuos distribuidos homogéneamente en el mismo número de tipos funcionales, pero en diferentes números de especies. T: número de tipos funcionales; S: número de especies; N: número total de individuos; $\sigma$ : diversidad funcional.

Case 2. 120 individuals equally distributed in same number of functional types, though in different number of species. T: number of functional types; S: number of tree species; N: total individual number;

\begin{tabular}{|c|c|c|c|}
\hline Subcasos & $\begin{array}{c}\text { Tipos } \\
\text { funcionales }\end{array}$ & $\begin{array}{c}\text { Especies } \\
\text { (Individuos) }\end{array}$ & $\sigma$ \\
\hline \multirow{6}{*}{$\begin{array}{l}\text { Caso 2.1: } T=3 \text {; } \\
S=6 ; N=120\end{array}$} & \multirow{2}{*}{ A } & $\mathrm{a}(20)$ & \multirow{6}{*}{54} \\
\hline & & b (20) & \\
\hline & \multirow{2}{*}{ B } & c (20) & \\
\hline & & $\mathrm{d}(20)$ & \\
\hline & \multirow{2}{*}{$\mathrm{C}$} & e (20) & \\
\hline & & $f(20)$ & \\
\hline \multirow{12}{*}{$\begin{array}{l}\text { Caso 2.2: } \mathrm{T}=3 ; \\
\mathrm{S}=12 ; \mathrm{N}=120\end{array}$} & \multirow{4}{*}{ A } & a (10) & \multirow{12}{*}{108} \\
\hline & & b (10) & \\
\hline & & c (10) & \\
\hline & & $\mathrm{d}(10)$ & \\
\hline & & a (10) & \\
\hline & $\mathrm{B}$ & b (10) & \\
\hline & D & c (10) & \\
\hline & & $\mathrm{d}(10)$ & \\
\hline & \multirow{4}{*}{$\mathrm{C}$} & a (10) & \\
\hline & & b (10) & \\
\hline & & c (10) & \\
\hline & & $\mathrm{d}(10)$ & \\
\hline
\end{tabular}
$\sigma$ : functional diversity. 
Cuadro 3. Caso 3120 individuos distribuidos heterogéneamente en las especies' y los tipos funcionales. T: número de tipos funcionales; $\mathrm{S}$ : número de especies; $\mathrm{N}$ : número total de individuos; $\sigma$ : diversidad funcional.

Case 3. 120 individuals heterogeneously distributed in species and functional types. T: number of functional types; $\mathrm{S}$ : number of tree species; $\mathrm{N}$ : total individual number; $\sigma$ : functional diversity.

\begin{tabular}{|c|c|c|c|}
\hline Subcasos & $\begin{array}{c}\text { Tipos } \\
\text { funcionales }\end{array}$ & $\begin{array}{c}\text { Especies } \\
\text { (Individuos) }\end{array}$ & $\sigma$ \\
\hline \multirow{6}{*}{$\begin{array}{c}\text { Caso 3.1: } \mathrm{T}=3 ; \\
\mathrm{S}=6 ; \mathrm{N}=120\end{array}$} & \multirow{2}{*}{ A } & a (30) & \multirow{6}{*}{48} \\
\hline & & b (10) & \\
\hline & $B$ & c (30) & \\
\hline & D & $\mathrm{d}(10)$ & \\
\hline & \multirow{2}{*}{$\mathrm{C}$} & e (30) & \\
\hline & & $\mathrm{f}(10)$ & \\
\hline \multirow{6}{*}{$\begin{array}{c}\text { Caso 3.2: } \mathrm{T}=3 ; \\
\mathrm{S}=6 ; \mathrm{N}=120\end{array}$} & A & a (20) & \multirow{6}{*}{36} \\
\hline & \multirow{2}{*}{ B } & b (20) & \\
\hline & & $\mathrm{c}(20)$ & \\
\hline & \multirow{3}{*}{$\mathrm{C}$} & $\mathrm{d}(20)$ & \\
\hline & & e (20) & \\
\hline & & $\mathrm{f}(20)$ & \\
\hline \multirow{6}{*}{$\begin{array}{c}\text { Caso 3.3: } T=3 ; \\
S=6 ; N=120\end{array}$} & A & $\mathrm{a}(40)$ & \multirow{6}{*}{40,5} \\
\hline & \multirow{2}{*}{ B } & b (30) & \\
\hline & & c (10) & \\
\hline & \multirow{3}{*}{$\mathrm{C}$} & $\mathrm{d}(20)$ & \\
\hline & & e (10) & \\
\hline & & $f(10)$ & \\
\hline
\end{tabular}

Diversidad funcional en el bosque semideciduo micrófilo. En el cuadro 4 se muestra la distribución de individuos, especies y tipos funcionales en sitios con diferente nivel de perturbación según Salmerón et al. (2016). Los tres tipos funcionales de respuesta a las perturbaciones previamente definidos, fueron encontrados tanto en sitios poco perturbados como en los medianamente y muy perturbados, por lo que la riqueza de tipos funcionales (T) en todos los sitios fue tres $(T=3)$. En los sitios muy perturbados se reportaron cuatro especies cobertoras con 1.261 individuos, cuatro especies colonizadoras con 112 individuos y una especie estabilizadora con un individuo. En los sitios medianamente perturbados se reportaron 12 especies cobertoras con 1.429 individuos, 11 especies colonizadoras con 1.922 individuos y 10 especies estabilizadoras con 221 individuos. En los sitios poco perturbados se reportaron 10 especies cobertoras con 1.425 individuos, nueve especies colonizadoras con 2.633 individuos y 11 especies estabilizadoras con 406 individuos.

En la figura 1 se pueden comparar las proporciones entre especies, individuos y abundancias relativas por tipos funcionales según los niveles de perturbación.
En el cuadro 4 se observa que para los sitios muy perturbados se obtuvo el menor valor de la diversidad funcional $(\sigma=1,56)$ mientras que para los sitios medianamente perturbados y poco perturbados, la diversidad funcional, aunque significativamente más alta que en los sitios muy perturbados, se mantuvo en valores próximos $(\sigma=26,33$ y $\sigma=28,65$, respectivamente).

En el cuadro 5 se presenta una comparación de los resultados obtenidos en el cálculo para los sitios con diferente nivel de perturbación, de los indicies de biodiversidad más comunes usados en la literatura y el índice de diversidad funcional $\sigma$.
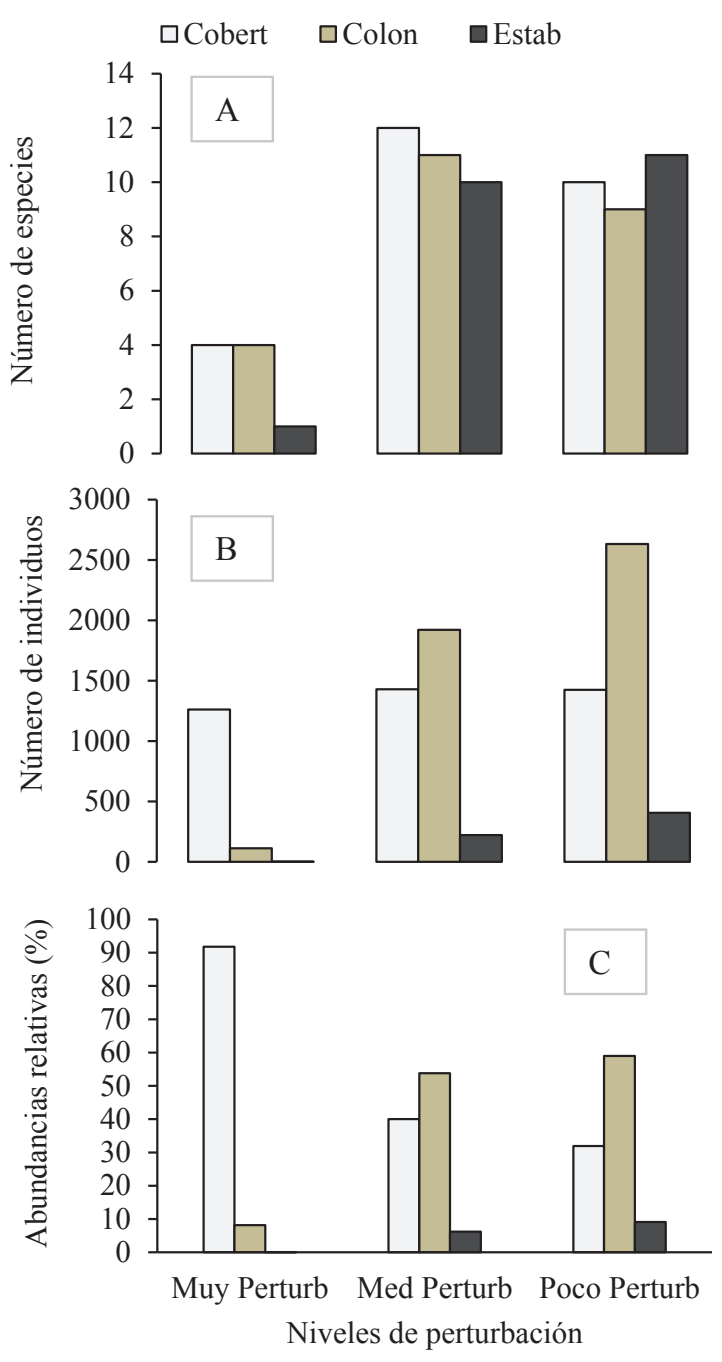

Figura 1. Riqueza de especies (A), abundancia absoluta (B) y abundancia relativa (C) por tipo funcionales y nivel de perturbación. Cobert: especies cobertoras. Colon: especies colonizadoras. Estab: especies estabilizadoras. Muy (Med, Poco) Perturb: Muy (Medianamente, Poco) perturbado.

Species richness (A), absolute abundance (B) and relative abundance (C), by functional type and disturbance level. Cobert: spread species. Colon: settler species. Estab: stabilizer species. Muy (Med, Poco) Disturbance: High (moderate, low) disturbed. 
Cuadro 4. Diversidad funcional para sitios sometidos a diferentes niveles de perturbación. $n_{i j:}$ número de individuos de la especie $\mathrm{i}$ del tipo funcional j. $S_{j}$ : número de especies en el tipo funcional j. $m_{j}$ : número de individuos en el tipo funcional j. T: número de tipos funcionales. $N$ : número total de individuos. Muy (Med, Poc) Prt: Muy (Medianamente, Poco) perturbado.

Functional diversity for site subject to different disturbance levels. $n_{i j}$ individuals number in the i species and j functional type $S_{i}$ : species number in the functional type j. $m_{j}$ : individual number in the functional type j. $T$ : functional types number. $N$ : total individuals number. Muy (Med, Poc) Prt: High (moderate, low) disturbed.

\begin{tabular}{|c|c|c|c|c|c|c|}
\hline \multirow{2}{*}{ Especies } & \multicolumn{3}{|c|}{ Muy Prt } & \multicolumn{2}{|l|}{ Med Prt } & Poc Prt \\
\hline & $\operatorname{Pr}$ & $n_{i j}$ & $\operatorname{Pr}$ & $n_{i j}$ & $\operatorname{Pr}$ & $n_{i j}$ \\
\hline \multicolumn{7}{|l|}{ Tipo funcional: cobertoras } \\
\hline Vachellia macracantha & 1 & 1.198 & 1 & 154 & 1 & 14 \\
\hline Senna atomaria & 1 & 56 & 1 & 36 & 1 & 15 \\
\hline Croton lucidus & 0 & 0 & 1 & 292 & 1 & 836 \\
\hline Colubrina elliptica & 0 & 0 & 1 & 656 & 1 & 157 \\
\hline Bursera simaruba & 0 & 0 & 1 & 98 & 1 & 242 \\
\hline Exostema caribaeum & 0 & 0 & 1 & 91 & 1 & 107 \\
\hline Tecoma stans & 1 & 4 & 1 & 41 & 1 & 37 \\
\hline Melochia tomentosa & 1 & 3 & 1 & 24 & 0 & 0 \\
\hline Zanthoxylum fagara & 0 & 0 & 1 & 16 & 0 & 0 \\
\hline Coulteria linnaei & 0 & 0 & 1 & 4 & 0 & 0 \\
\hline Plumeria obtusa L. & 0 & 0 & 1 & 15 & 1 & 9 \\
\hline Tabebuia myrtifolia & 0 & 0 & 0 & 0 & 1 & 3 \\
\hline Thouinia trifoliata & 0 & 0 & 1 & 2 & 1 & 5 \\
\hline Total de cobertoras & $S_{j}=4$ & $m_{j}=1.261$ & $S_{j}=12$ & $m_{j}=1.469$ & $S_{j}=10$ & $m_{j}=1.425$ \\
\hline \multicolumn{7}{|l|}{ Tipo funcional: colonizadoras } \\
\hline Rauwolfia ligustrina & 1 & 7 & 0 & 0 & 0 & 0 \\
\hline Bourreria virgata & 1 & 29 & 1 & 112 & 0 & 0 \\
\hline Randia aculeata & 1 & 54 & 1 & 1.126 & 0 & 0 \\
\hline Erythroxylum havanense & 1 & 29 & 1 & 212 & 0 & 0 \\
\hline Eugenia monticola & 0 & 0 & 1 & 67 & 1 & 228 \\
\hline Adelia ricinella & 0 & 0 & 1 & 83 & 1 & 26 \\
\hline Zanthoxylum pistaciifolium & 0 & 0 & 1 & 21 & 1 & 3 \\
\hline Diospyros grisebachii & 0 & 0 & 1 & 78 & 1 & 90 \\
\hline Amyris elemifera & 0 & 0 & 1 & 11 & 1 & 26 \\
\hline Sideroxylon salicifolium & 0 & 0 & 1 & 20 & 1 & 77 \\
\hline Gymnanthes lucida & 0 & 0 & 1 & 82 & 1 & 987 \\
\hline Coccoloba diversifolia & 0 & 0 & 1 & 110 & 1 & 534 \\
\hline Heterosavia bahamensis & 0 & 0 & 0 & 0 & 1 & 662 \\
\hline Total de colonizadoras & $S_{j}=4$ & $m_{j}=119$ & $S_{j}=11$ & $m_{j}=1.922$ & $S_{j}=9$ & $m_{j}=2.633$ \\
\hline \multicolumn{7}{|l|}{ Tipo funcional: estabilizadoras } \\
\hline Erithalis fruticosa & 0 & 0 & 1 & 97 & 1 & 74 \\
\hline Eugenia cowelli & 0 & 0 & 1 & 46 & 1 & 8 \\
\hline Guettarda elliptica & 0 & 0 & 1 & 11 & 0 & 0 \\
\hline Eugenia iteophylla & 0 & 0 & 1 & 8 & 1 & 8 \\
\hline Pseudocarpidium avicennioides & 0 & 0 & 1 & 8 & 0 & 0 \\
\hline Picrodendron baccatum & 0 & 0 & 0 & 0 & 1 & 12 \\
\hline Guettarda cueroensis & 0 & 0 & 1 & 21 & 1 & 23 \\
\hline Cordia gerascanthus & 0 & 0 & 1 & 3 & 1 & 21 \\
\hline Crossopetalum rhacoma & 0 & 0 & 1 & 15 & 1 & 164 \\
\hline Pseudocarpidium ilicifolium & 0 & 0 & 1 & 10 & 1 & 56 \\
\hline Erythroxylum rotundifolium & 1 & 1 & 1 & 2 & 1 & 26 \\
\hline Banara minutiflora & 0 & 0 & 0 & 0 & 1 & 7 \\
\hline Citharexylum spinosum & 0 & 0 & 0 & 0 & 1 & 7 \\
\hline \multirow[t]{2}{*}{ Total de estabilizadoras } & $S_{j}=1$ & $m_{j}=1$ & $S_{j}=10$ & $m_{j}=221$ & $S_{j}=11$ & $m_{j}=406$ \\
\hline & $T=3$ & $N=1.941$ & $T=3$ & $N=3.020$ & $T=3$ & $N=4.472$ \\
\hline Diversidad funcional & \multicolumn{2}{|c|}{$\sigma=1,56$} & \multicolumn{2}{|c|}{$\sigma=26,33$} & \multicolumn{2}{|c|}{$\sigma=28,65$} \\
\hline
\end{tabular}


Cuadro 5. Comparación entre índices de biodiversidad y el índice $\sigma$ en sitios con diferentes niveles de perturbación. S: Riqueza de especies. $\lambda$ : Dominancia según el índice de Simpson. $C_{i n v}$ : Diversidad según el inverso de Simpson. H: índice de ShannonWeaver. $\sigma$ : diversidad funcional según el índice $\sigma$.

Comparison between conventional diversity index and $\sigma$ index in sites with different disturbance levels. S: Species richness. $\lambda$ : Dominance as Simpson index. $\mathrm{C}_{\text {inv }}$ : Diversity as Simpson inverse index. H: Diversity as Shannon-Weaver index. $\sigma$ : Functional diversity as $\sigma$ index.

\begin{tabular}{lccccc}
\hline Niveles de perturbación & $\mathrm{S}$ & $\lambda$ & $\mathrm{C}_{\text {inv }}$ & $\mathrm{H}$ & $\sigma$ \\
\hline Muy perturbados & 9 & 0,54 & 1,85 & 0,95 & 1,56 \\
Medianamente perturbados & 33 & 0,15 & 6,59 & 2,22 & 26,30 \\
Poco perturbados & 30 & 0,13 & 7,67 & 2,40 & 28,65 \\
\hline
\end{tabular}

\section{DISCUSIÓN}

El parámetro $\rho$ en la ecuación [13] representa un indicador de la dominancia de las especies $i$ y de los tipos funcionales $i$ y su valor es la probabilidad de que al elegir dos pares de individuos exista una coincidencia en la especie para un individuo en cada par y en el tipo funcional al que corresponde dicha especie para el otro individuo de cada par, aunque estos últimos puedan corresponder a cualquiera de las especies incluidas dentro del tipo funcional dado.

La consideración de la diversidad como el inverso de la dominancia concuerda con el criterio expuesto por Feinsinger (2004).

El índice propuesto es más sensible a cambios en la equidad del número de individuos entre diferentes tipos funcionales que entre especies (ver variaciones en los casos 3.1, 3.2 y 3.3 en el cuadro 3). En general, la diversidad funcional será mayor cuando las comunidades tengan más tipos funcionales ( $\sigma$ en $1.3>\sigma$ en 1.2), más especies por tipos funcionales ( $\sigma$ en $2.2>\sigma$ en 2.1) y más equitativa distribución de las especies entre los tipos funcionales.

Puede verificarse, de los casos analizados, que el mayor valor posible de la diversidad funcional, según el índice propuesto, en una comunidad de $\mathrm{S}$ especies y $\mathrm{T}$ tipos funcionales es el valor $\mathrm{ST}^{2}$ y este se alcanza cuando los individuos se encuentran homogéneamente distribuidos en las especies y estas en los tipos funcionales.

Es evidente que dos comunidades con el mismo valor para la diversidad de especies pueden tener diferentes valores de diversidad funcional. Por ejemplo, los casos $2.1 \mathrm{y}$ 3.2 tienen el mismo número de especies y el mismo número de individuos por especies, por lo que el valor de cualquier índice de biodiversidad resultará el mismo, sin embargo, el índice de diversidad funcional arroja dos valores diferentes, debido a que este último brinda información adicional referida a cómo se distribuyen estas especies y los individuos en los tipos funcionales que componen la comunidad.
Menos evidente puede resultar el hecho que se observa al comparar los casos 3.2 y 3.3. Ahora la diversidad calculada mediante el coeficiente inverso de Simpson, según la ecuación [3], es mayor para el caso $3.2\left(C_{i n v}=6\right)$ que para el caso $3.3\left(\mathrm{C}_{\mathrm{inv}}=4,5\right)$ mientras que los valores de $\sigma$ arrojan, respectivamente, 36 para el caso 3.2 y 40,5 para el caso 3.3. Es decir, puede darse el caso de que una comunidad con una diversidad específica dada, según el índice inverso de Simpson, tenga menor diversidad funcional según el índice $\sigma$, que otra con menor diversidad específica según el mismo índice inverso de Simpson. Dicho de otro modo, no existe una relación proporcional entre la diversidad funcional calculada por el índice que se propone y la diversidad específica, calculada por los índices comúnmente usados, particularmente el inverso de Simpson.

Puede verificarse, de los casos analizados, que el mayor valor posible de la diversidad funcional, según el índice propuesto, en una comunidad de $\mathrm{S}$ especies y $\mathrm{T}$ tipos funcionales, es el valor $\mathrm{ST}^{2}$ y este se alcanza cuando los individuos se encuentran homogéneamente distribuidos en las especies y estas en los tipos funcionales.

Al comparar el índice de diversidad funcional $\sigma$, con el índice de Rao (Leps et al. 2006) la diferencia sustancial es que, en el caso del índice de Rao no se determinan inicialmente tipos funcionales. Para ese índice todas las especies entre las que exista una determinada "distancia funcional" $\mathrm{dij}>0$, atendiendo a un rasgo funcional dado, ya son consideradas distintas funcionalmente. El índice de Rao es también insensible a la redundancia funcional, es decir no varía si los individuos con el mismo rasgo funcional pertenecen a la misma o a diferentes especies.

Respecto al índice MFAD (Casanoves et al. 2011), el índice $\sigma$ coincide en la determinación de tipos funcionales inicialmente. Sin embargo, en el MFAD, a partir de que los individuos de las diferentes especies sean incluidos en un determinado grupo funcional, son considerados como una misma especie, y por lo tanto ese índice es también insensible a la redundancia de estas en esos tipos funcionales, lo que no ocurre en el índice $\sigma$.

Resulta innegable la utilidad del índice propuesto $(\sigma)$ para estudios que vinculen la diversidad funcional a la integridad de los ecosistemas, puesto que la redundancia funcional ha sido reconocida como un aspecto de importancia para el mantenimiento de la integridad de los sistemas ecológicos (Petchey y Gaston 2009, Thompson et al. 2009).

Este estudio coincide, en casi todos los criterios expuestos por Mason et al. (2003) respecto a las características que debe cumplir cualquier índice de diversidad funcional, particularmente en lo referido a que debe ser sensible a la abundancia de las especies, pero no coincide en ser insensible al método de construcción de los tipos funcionales y al número de especies. La diversidad funcional del sistema depende de cuál aspecto de la funcionalidad de este se está analizando. En efecto, los tipos funcionales definidos dependen también, según Lavorel (1999) y Díaz 
y Cabido (2001) con quienes se coincide, del aspecto de la funcionalidad tenido en cuenta, y por lo tanto el índice de diversidad funcional constituye un atributo que caracteriza a este aspecto de la funcionalidad analizado en el sistema objeto de estudio.

La diversidad funcional calculada por el índice $\sigma$ corresponde, en este caso, a la diversidad de tipos de respuesta a las perturbaciones en el bosque semideciduo de la reserva. Si los tipos funcionales de los que se parte (Salmerón et al.2016) hubieran sido definidos desde otro enfoque funcional, muy probablemente el valor de la diversidad funcional hubiera sido otro.

Si se analiza a la luz de la clasificación en diferentes categorías realizada por Mouchet et al. (2010) para los índices de diversidad funcional, el índice $\sigma$ es un índice de uniformidad, en efecto, a medida que los individuos se distribuyan de manera más equitativa en las especies y estas en los tipos funcionales, mayor es el valor del índice.

Los resultados obtenidos para el índice $\sigma$ en el bosque semideciduo micrófilo de la Reserva Ecológica Siboney Juticí indican, por lo tanto, que en los sitios poco perturbados las especies y los individuos están más homogéneamente distribuidos entre los tres tipos funcionales que en los sitios medianamente perturbados, y en estos últimos mejor que en los sitios muy perturbados. Esta diferencia resulta difícil de evaluar a simple vista a partir de la figura 1, fundamentalmente para sitios poco y medianamente perturbados, pero se determina fácilmente cuando se aplica el índice $\sigma$ y se tiene en cuenta el significado del mismo.

Debe tenerse en cuenta que en el caso que se analiza no se considera ningún sitio exento de perturbaciones. Los sitios poco perturbados, tienen aún un nivel de perturbación originado por actividades humanas realizadas en algún momento de su devenir histórico. Esto puede ser la causa de la proximidad de los valores de diversidad funcional entre estos y los medianamente perturbados. Estos aspectos deben ser estudiados con más profundidad en el futuro.

Los cambios en la riqueza de especies al variar los niveles de perturbación coinciden con lo reportado en la literatura para otras latitudes (Mishra et al. 2004, Sahu et al. 2008), sobre todo en lo referente a la drástica disminución de la riqueza de especies si aumentan las perturbaciones, tal como expresan Mishra et al. (2004), aunque se contradicen los resultados obtenidos por Kumar y Ram (2005) en este sentido, quienes encontraron la mayor riqueza de especies en los sitios más perturbados.

Para el bosque semideciduo micrófilo analizado, aunque en todos los sitios están presentes los tres tipos funcionales definidos, en los sitios medianamente y poco perturbados, no solo hay mayor riqueza de especies sino que hay más especies por cada tipo funcional (y por lo tanto mayor redundancia funcional) $\mathrm{y}$, en consecuencia, la resiliencia de estos frente a las perturbaciones externas (Thompson et al. 2009) es mayor que en los sitios muy perturbados. Esta afirmación tampoco puede ser realizada a partir de los índices de diversidad convencionales (ecuaciones 3, 4 y 5, y cuadro 5). Tampoco pudiera ser deducida completamente a partir de índices de diversidad funcional que no reconocen la redundancia funcional, como el de Rao y el MFAD (Leps et al. 2006, Casanoves et al. 2011).

Para Petchey y Gastón (2009), la pérdida de especies dentro de un tipo funcional con más de una especie no implica una disminución en la diversidad funcional del ecosistema. Bajo el enfoque que aquí se propone, mediante el cálculo del índice $\sigma$, esto no ocurre: la pérdida de una especie (cobertora, colonizadora o estabilizadora) implica siempre una disminución de la diversidad funcional y de la capacidad de respuesta del sistema. La afirmación de Petchey y Gaston (2009) se debe a que esos autores utilizan la riqueza de tipos funcionales como un índice de diversidad funcional.

\section{CONCLUSIONES}

El índice propuesto permite calcular la diversidad funcional considerando la redundancia de las especies y aporta información útil sobre la resiliencia del bosque frente a perturbaciones tanto antrópicas como naturales.

El cálculo del índice en el bosque semideciduo micrófilo de la Reserva Ecológica Siboney Juticí muestra que los estados poco perturbados y medianamente perturbados tienen mayor diversidad funcional que los muy perturbados y por lo tanto poseen mayor resiliencia frente a las posibles perturbaciones.

Perturbaciones que impliquen mayor disminución en la diversidad funcional, disminuyen la posibilidad de bosque para recuperarse una vez que haya cesado la perturbación.

El manejo orientado a mantener la diversidad funcional en valores similares a los obtenidos en los sitios poco perturbados, tomando como referencia el cálculo con el índice $\sigma$, constituye una garantía para mantener la complejidad estructural y, por lo tanto, la integridad del bosque y su capacidad de respuesta frente a un régimen cambiante de perturbaciones naturales y antrópicas.

\section{REFERENCIAS}

Casanoves F, L Pla, J Di Rienzo, S Díaz. 2011. FDiversity: a software package for the integrated analysis of functional diversity. Methods in Ecology and Evolution 2(3): 233-237.

Díaz S, F Quétier, MD Cáceres, SF Trainor, N Pérez-Harguindeguy, MS Bret-Harte, B Finegan, M Peña-Claros, L Poorter. 2011. Linking functional diversity and social actor strategies in a framework for interdisciplinary analysis of nature's benefits to society. PNAS 108 (3): 895-900.

Díaz S, M Cabido. 2001. Vive la différence: plant functional diversity matters to ecosystem processes. Trends in Ecology and Evolution 16 (11): 646-655.

Feinsinger P. 2004. El diseño de estudios de Campo para la conservación de la Biodiversidad. Santa Cruz de la Sierra, Bolivia. Editorial FAN. 242 p.

Fyllas NM, CA Quesada, J Lloyd. 2012. Deriving Plant Functional Types for Amazonian forests for use in vegetation dyna- 
mics models. Perspectives in Plant Ecology, Evolution and Systematics 14(2): 97-110.

Gillison AN, DE Bignell, RW Kenneth, ECM Brewer, DT Fernández, JD Sheil, $\mathrm{PH}$ May, $\mathrm{AD}$ Watt, R Constantino, EG Couto, K Hairiah, P Jepson, P Agus, I Maryanto, G Neto, M Noordwijk, EA Silveira, FX Susilo, SA Vosti, PC Nunes. 2013. Plant functional types and traits as biodiversity indicators for tropical forests: two biogeographically separated case studies including birds, mammals and termites. Biodiversity and Conservation 22(9): 1909-1930. DOI 10.1007/ s10531-013-0517-1.

Gondard H, S Jauffret, J Aronson, S Lavorel. 2003. Plant functional types: a promising tool for management and restoration of degraded lands. Applied Vegetation Science 6(2): 223-234.

Gunderson LH. 2000. Ecological resilience in theory and application. Annual Review for Ecological System 31(1): 425-439.

Kooyman R, M Rossetto. 2008. Definition of plant functional groups for informing implementation scenarios in resourcelimited multi-species recovery planning. Biodiversity and Conservation 17(12): 2917-2937.

Kumar A, J Ram. 2005. Anthropogenic disturbances and plant biodiversity in forests of Uttaranchal, central Himalaya. Biodiversity and Conservation 14(2): 309-331.

Lavorel S. 1999. Ecological diversity and resilience of Mediterranean vegetation to disturbance. Diversity and Distributions 5(1-2): 3-13.

Leps J, F de Bello, S Lavorel, S Berman. 2006. Quantifying and interpreting functional diversity of natural communities: practical considerations matter. Preslia 78(4): 481-501.

Mason NWH, K MacGillivray, JB Steel, JB Wilson. 2003. An index of functional diversity. Journal of Vegetation Science 14(4): 571-578.

Mishra BP, OP Tripathi, RS Tripathi, HN Pandey. 2004. Effects of anthropogenic disturbance on plant diversity and community structure of a sacred grove in Meghalaya, northeast India. Biodiversity and Conservation 13(2): 421-436.
Moreno CE. 2001. Métodos para medir la biodiversidad. Zaragoza, España. M\&T-Manuales y Tesis SEA. 84 p.

Mouchet MA, S Villeger, WH Mason, D Mouillot. 2010. Functional diversity measures: an overview of their redundancy and their ability to discriminate community assembly rules. Functional Ecology 24(4): 867-876.

Petchey OL, KJ Gaston. 2009. Effects on ecosystem resilience of biodiversity, extinctions, and the structure of regional species. Theoretical Ecology 2(3):177-187.

Quétier F, S Díaz, The Diversus collaborative research network (Diversus). 2008. The effects of functional biodiversity on ecosystem processes, ecosystem services and sustainability: An interdisciplinary approach. GLP NEWS 3: 28-31.

Reyes J, F Acosta. 2005. Vegetación Terrestre en Cuba: Siboney Juticí. Rapid Biological Inventories, Report 10. Chicago, USA. The Field Museum. 73 p.

Sahu PK, R Sagar, JS Singh. 2008. Tropical forest structure and diversity in relation to altitude and disturbance in a Biosphere Reserve in central India. Applied Vegetation Science 11(4): 461-470.

Salmerón A, A González, G Geada. 2016. Tipos funcionales de plantas según su respuesta a las perturbaciones en un bosque semideciduo micrófilo costero de Cuba Oriental. Bosque 37(1): 131-141. DOI: 10.4067/S071792002016000100013.

Thompson I, B Mackey, S McNulty, A Mosseler. 2009. Forest Resilience, Biodiversity, and Climate Change. A synthesis of the biodiversity/resilience/stability relationship in forest ecosystems. Secretariat of the Convention on Biological Diversity, Montreal. Technical Series 43: $67 \mathrm{p}$.

Uriarte M, NG Swenson, RL Chazdon, LS Comita, WJ Kress, D Erickson, J Forero- Montan, JK Zimmerman, J Thompson. 2010. Trait similarity, shared ancestry and the structure of neighbourhood interactions in a subtropical wet forest: implications for community assembly. Ecology Letters 13(12): 1503-1514. 